\title{
Transmitter role of the luminal uterine epithelium in the induction of decidualization in rats
}

\author{
B. Lejeune, J. Van Hoeck and F. Leroy \\ Laboratory of Gynaecology \& Research on Human Reproduction, St Pierre Hospital, \\ Free University Brussels, Rue Haute 322, 1000 Brussels, Belgium
}

\begin{abstract}
Summary. Ovariectomized rats were given hormonal treatment mimicking progestational ovarian secretions. At maximal uterine sensitivity, the luminal epithelium was squeezed out of one or both horn(s) transected at the isthmus. Simultaneous bilateral scratching and saline injection induced virtually no response in stripped horns while contralateral intact horns exhibited a maximal decidual reaction (DCR). The luminal epithelium regenerated after ablation and a $65 \%$ DCR was again elicited after 9 days. The inability of de-epitheliated horns to decidualize was not overcome by intraluminal injections of the supernatant or sediment of the $60000 \mathrm{~g}$ homogenate of epithelium, PGF-2 $\alpha$, PGE-2, arachidonic acid or histamine. Detachment of the epithelium without removal also prevented DCR induction. These results indicate that the luminal epithelium is an obligatory transmitter of the stimulus to DCR and cannot be by-passed by trauma. Release of the appropriate epithelial message to the stroma requires local preservation of membrane relationships between both tissues.
\end{abstract}

\section{Introduction}

In rats and mice, at Day 5 of pregnancy, the uterine surface epithelium appears still intact when the decidual cell reaction (DCR) is already developing. Genetically programmed epithelial cell degeneration does not start before Day 6 (Finn \& Porter, 1975). Therefore, the physiological signal from the blastocysts that initiates decidualization must in some way be transmitted through the epithelial layer.

Uterine trauma, such as scratching or crushing, elicits a DCR in pseudopregnant animals or in ovariectomized females adequately sensitized with ovarian steroids, but whereas a normal or oil-induced DCR requires previous intervention of small amounts of oestrogen (Finn, 1977), traumatic deciduomata can also be obtained in animals treated solely with progesterone (De Feo, 1967). It has therefore been proposed that one of the roles of 'nidatory' oestrogen is to act upon epithelial cells by enabling them to transmit the embryonic message, whereas trauma would by-pass this step and affect the stroma directly (Finn \& Martin, 1974). However, traumatic procedures would only be efficient if epithelial cells are being damaged (Fainstat. 1963; Psychoyos, 1973). Also when the epithelium is selectively frozen without injury to the underlying stroma, the DCR cannot be elicited by mere scratching (Ferrando \& Nalbandov, 1968). These results suggest that the surface epithelium is a compulsory intermediate in the induction of a DCR by trauma. The aims of the present work were to determine which of the foregoing interpretations for the role of the epithelium is correct and to investigate which substance(s) could play the role of transmitter(s) between epithelial and stromal cells in the mechanism of decidualization. 


\section{Materials and Methods}

Ovariectomy of virgin Wistar rats (body weight 150-200 g) was performed under general anaesthesia ( $2 \%$ tribromoethanol $+1 \%$ amylene hydrate: Aldrich Europe, Beerse, Belgium). After 7 days. hormone treatment was given to mimic the progestational ovarian secretions: i.e. $500 \mathrm{ng}$ oestradiol-17 (Sterop. Brussels) for 2 days, 2 days of rest, and then a daily dose of $3 \mathrm{mg}$ progesterone (Sterop. Brussels) for 3 days. On the last day, $50 \mathrm{ng}$ oestradiol-17 $\beta$ were also injected. The hormones were injected subcutaneously in arachis oil.

At maximal uterine sensitivity (i.e. $15 \mathrm{~h}$ after the last injection of oestrogen), laparotomy was performed under general anaesthesia and both uterine horns were transected at the isthmus. This allowed the luminal epithelium to be removed in one sheet by gentle longitudinal squeezing of one or both cornua. This method for in-vivo ablation was derived from that used by Bitton-Casimiri, Rath \& Psychoyos (1977) on dissected uteri. Histological examination showed that the tissue removed was pure epithelium, with the remaining tissue as intact stroma and glands (see PI. 1. Figs 1 and 2).

Different treatments were applied to the uterine horns immediately before or after uni- or bilateral removal of the epithelium. In each animal, one side was treated in such a manner as to serve as internal control.

Group 1. Both uterine horns were longitudinally scratched immediately before (Group 1a) or after (Group Ib) the removal of the epithelium from the left horn and $30 \mu \mathrm{PBS}(\mathrm{pH} \mathrm{7 \cdot 2)}$ (Pearse, 1968) were injected. Intrauterine injection was performed after placing a transverse ligature on the distal end of the horn to permit ligation as the needle was withdrawn, thus preventing leakage of the injected fluid.

Group 2. Both horns were injected with $30 \mu \mathrm{l}$ arachis oil. After $4 \mathrm{~h}$ the epithelium was removed from the left side only.

Group 3. The left horn was thoroughly crushed with a large pair of forceps along its entire length while the right one was transversely pinched at two points approximately at the ends of the median third of the horn.

Group 4. The right horn was squeezed but the epithelial sheet was not removed. Epithelial ablation was completed on the other side.

Group 5. The epithelium was removed from the left horn. while that of the right was left intact. After 9 days, the hormonal treatment described above was given and both horns were scratched bilaterally.

Group 6. The epithelium was squeezed out. or detached without removal from the lumen, in only the upper or the lower half of the experimental horn. Both horns were then left untouched or longitudinally scratched. There were therefore 6 different treatments applied to 23 animals, i.e. 46 horns (see details in 'Results'), in this group.

Group 7. The epithelium was removed from both horns and then $30 \mu \mathrm{PBS}$ were injected on the right side, while histamine chlorhydrate ( $1 \mathrm{mg}$ in $30 \mu \mathrm{l} \mathrm{PBS}$ ) (Sigma, St Louis, Missouri, U.S.A.) was injected intraluminally into the left horn.

Group 8. The right horn was longitudinally scratched, while the left one was injected, after removal of the epithelium. with $100 \mu \mathrm{g}$ arachidonic acid (Sigma) in $30 \mu \mathrm{l}$ PBS, $100 \mu \mathrm{g}$ prostaglandin (PG) E-2 (Sigma) in $30 \mu \mathrm{l} \mathrm{PBS,} \mathrm{or} 100 \mu \mathrm{g}$ PGF-2 $\alpha$ (Sigma) in $30 \mu \mathrm{l} \mathrm{PBS.}$

Group 9. Rats were hormonally treated as indicated above. At maximal uterine sensitivity, 18 animals were killed and the uterine epithelium was squeezed out of the dissected uteri. The pooled epithelial tissue was homogenized in $500 \mu \mathrm{l}$ PBS (pH 7.2) and centrifuged at $60000 \mathrm{~g}$ for $1 \mathrm{~h}$. The uterine epithelium was removed from both horns of rats identically primed with steroids and $30 \mu \mathrm{l}$ of the supernatant (Group 9a) or of the sediment resuspended in $30 \mu \mathrm{l}$ PBS (Group 9b) from the homogenized epithelium were injected into the left horn. The other side was treated with $30 \mu \mathrm{l}$ PBS as a control. Rats in Groups 9c and 9d were treated similarly except that the donor rats were given an intraluminal injection of arachis oil before the isolation and homogenization of the epithelium. 
In all groups, the animals were treated with $2 \mathrm{mg}$ progesterone plus $200 \mathrm{ng}$ oestradiol-17 $\beta$ per day given subcutaneously in oil for 4 days after the uterine treatment, starting on the same day. The rats were then killed and the DCR assessed by weighing the uterine horns.

\section{Results}

The results are summarized in Table 1.

Although submitted to the same stimuli (i.e. scratching and injection of PBS) as the intact control side, de-epitheliated horns were incapable of producing a DCR (Groups 1a and 1b), even when the ablation was carried out after scratching (Group 1a). Decidualization was also prevented when the epithelium was removed $4 \mathrm{~h}$ after oil instillation (Group 2). Totally crushed horns (Group 3) were still capable of producing a DCR of the same magnitude as that in the control, pinched horn. Detachment of the epithelium without removal was sufficient to prevent the occurrence of the DCR (Group 4).

Table 1. The effects of various treatments (see text) on the induction of the decidual cell reaction on rats

\begin{tabular}{cccc}
\hline & & \multicolumn{2}{c}{ Mean \pm s.e.m. uterine weight (mg) } \\
\cline { 3 - 4 } Group & $\begin{array}{c}\text { No. of } \\
\text { rats }\end{array}$ & Control horn (right) & Experimental horn (left) \\
\hline la & 9 & $1426 \pm 129$ & $140 \pm 19^{* *}$ \\
lb & 6 & $480 \pm 123$ & $90 \pm 9 \cdot 4^{*}$ \\
2 & 8 & $640 \pm 92$ & $167 \pm 20^{* *}$ \\
3 & 6 & $1200 \pm 230$ & $1190 \pm 250$ \\
4 & 7 & $221 \pm 37$ & $207 \pm 44$ \\
5 & 9 & $874 \pm 30$ & $555 \pm 59$ \\
6 & 23 & $($ see Pl. 1. Fig. 4$)$ & (see Pl. 1, Fig. 4) \\
7 & 6 & $198 \pm 20$ & $191 \pm 34$ \\
$8 \mathrm{a}$ & 6 & $754 \pm 80$ & $105 \pm 8 \cdot 2^{* *}$ \\
$8 \mathrm{~b}$ & 6 & $647 \pm 118$ & $170 \pm 33^{*}$ \\
$8 \mathrm{c}$ & 6 & $601 \pm 88$ & $138 \pm 6 \cdot 5^{* *}$ \\
$9 \mathrm{a}$ & 10 & $263 \pm 51$ & $297 \pm 57$ \\
$9 \mathrm{~b}$ & 14 & $203 \pm 25$ & $169 \pm 26$ \\
$9 \mathrm{c}$ & 4 & $212 \pm 55$ & $102 \pm 20$ \\
$9 \mathrm{~d}$ & 3 & $143 \pm 40$ & $140 \pm 35$ \\
\hline
\end{tabular}

Values significantly different from those of the control horn: $* P<0.01, * * P<0.001$ (Student's $t$ test).

In Group 5 the DCR response in the experimental horn was $65 \%$ of that in the control horn, showing that sensitive and reactive tissue was present after the 9-day rest (see Pl. 1, Fig. 3).

Detachment of the epithelium in the upper or lower half of a horn (Group 6) precluded a DCR in this treated region but a massive response in the adjacent untouched segment occurred in 21 of the 23 animals (PI. 1, Fig. 4). The detailed results for the subgroups were as follows. Of the 10 animals for which the epithelium was detached from the upper half of the experimental horn, 8 showed decidualization in the intact lower half and 2 had no deciduomata. The control horn was not scratched and no DCR was observed. When the epithelium was removed from the upper half of the experimental horn, all 4 animals showed a DCR in the intact lower half. The control horn was not scratched and showed no DCR. If the epithelium was removed from the upper half of the experimental horn which was then scratched along its total length, a DCR occurred in the lower half in all 4 animals. Removal of the epithelium from the lower half of the experimental horn and scratching of the entire horn induced decidualization in the upper half in all 5 animals, while scratching of the control horn induced a massive DCR in all rats. 
None of the treatments of the experimental horns in Groups 7,8 or 9 was able to induce a DCR.

\section{Discussion}

Failure to induce decidualization after removal of the luminal epithelium (Group 1) and the recovery of this ability after regeneration (Pl. 1, Fig. 3) of this tissue (Group 5) indicate that the epithelium plays the role of an obligatory transducer which cannot be by-passed in the mechanism of decidual induction. Since squeezing of the uterus might have caused histologically unapparent damage to the stromal cells, total longitudinal crushing of one horn was tested; in spite of the extensive tissue damage, the DCR was equivalent to that in the control horn. The unresponsiveness of de-epitheliated horns is therefore not related to stromal cell destruction provoked by the method of epithelial removal.

Deciduomata can be induced by electrical stimulation of uterine horns in receptive animals (Krebiehl, 1937; Ansbro \& Schwartz, 1965). Therefore, a possible way for epithelial cells to intervene, could be by a rapid electrical phenomenon, such as membrane depolarization occurring in response to direct epithelial stimulation. However, such a mechanism, if involved at all, cannot be solely responsible for decidual induction, since removal of the epithelium prevented the DCR, regardless of whether epithelial excision was performed immediately before or after uterine scratching (Groups $1 \mathrm{a}$ and $1 \mathrm{~b}$ ). The fact that two narrow pinches gave a massive response, extending to the entire length of the horn, would suggest transmission of some signal from damaged cells along the intact epithelial layer. Such a conclusion is also consistent with results from Group 6.

A different or complementary explanation would be the release of deciduogenic substances from the epithelium towards the stroma. Such a phenomenon would occur through apical membrane stimulation and/or damage of epithelial cells. It could correspond either to the liberation of pre-existing deciduogenic factors or to the induction of their synthesis before release. For induction by oil or an embryo, such a mechanism would also involve changes in basal permeability. Exocytosis at the basal pole of the epithelial layer of material pinocytosed from the lumen has been reported (Vokaer \& Leroy, 1962). Our experiments with epithelial extracts (Group 9) from stimulated or unstimulated donor uteri, show that (i) deciduogenic substances are apparently not stored within the epithelium (Groups 9a and 9b), and/or (ii) the amounts of such compounds eventually produced within $4 \mathrm{~h}$ after stimulation are not effective (Groups $9 \mathrm{c}$ and 9d). An appropriate epithelial message is clearly not reaching the stroma within $4 \mathrm{~h}$ after oil injection (Group 2).

The literature provides evidence that PGs could be involved in the mechanism of decidualization. In rabbits, intrauterine implants containing PGE-2, PGF-2 $\alpha$ or arachidonic acid elicit a prominent DCR (Hoffman, Strong, Davenport \& Frolich, 1977). Indomethacin (an

\section{PLATE 1}

Fig. 1. The progestational uterus of a rat after mechanical removal of the surface epithelium in vivo.

Fig. 2. Part of the excised epithelium showing the mutual apical adhesion of cells.

Fig. 3. Regenerating uterine epithelium 4 days after removal of the surface epithelium $\left(\left[{ }^{3} \mathrm{H}\right]\right.$ thymidine autoradiography).

Fig. 4. Typical effect on the distribution of the DCR after partial detachment or removal of the uterine epithelium (Group 6). Horns A1 and B2 are scratched control horns with a massive DCR. De-epithelization of upper (Horn A2) and lower (Horn B2) halves permits a DCR only in the intact adjacent segments. Epithelial ablation (Horn C2) or detachment (Horn D2) in the upper half of the left horn results in a massive DCR in the intact lower segment without extension to the untouched contralateral horns (C1 and D1). 


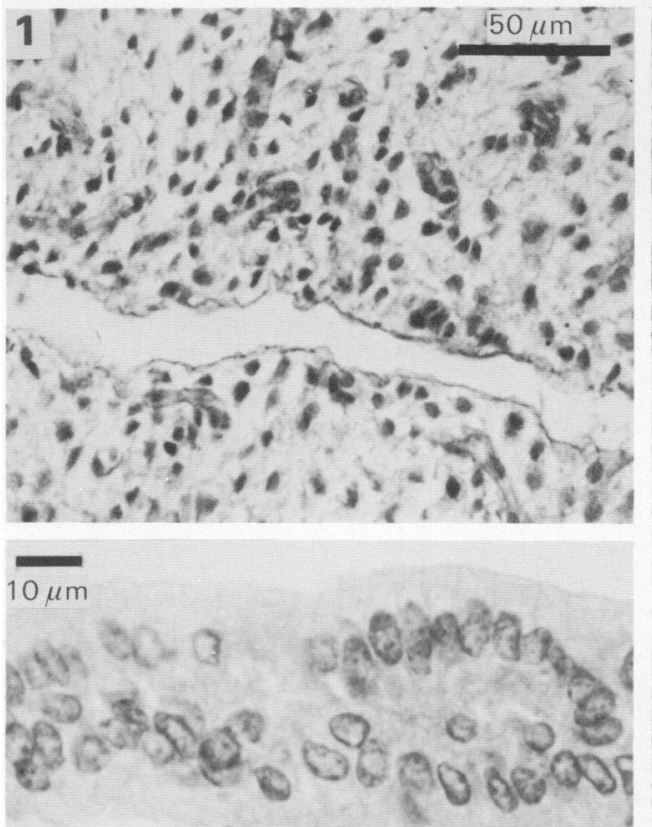

2

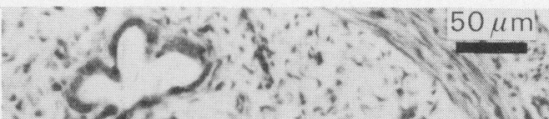

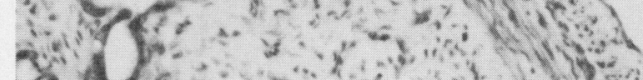

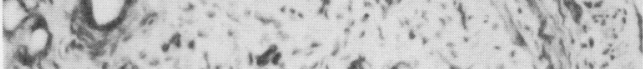

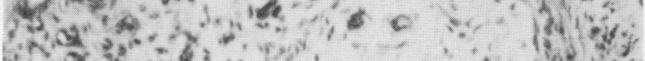

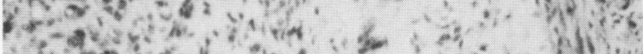

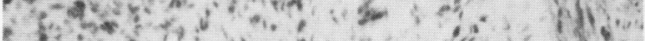

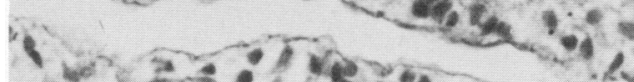

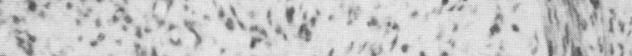

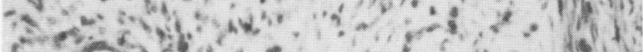

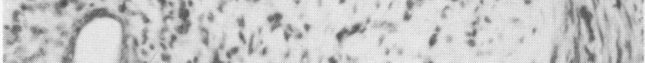

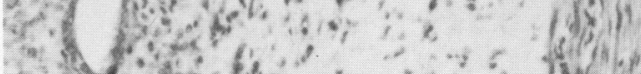

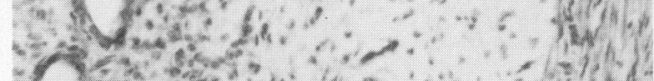

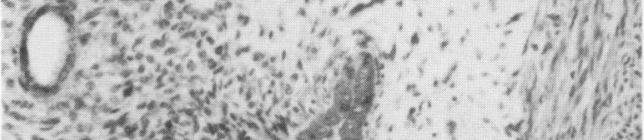

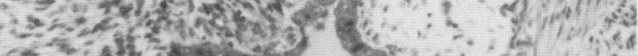

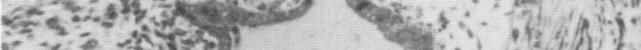

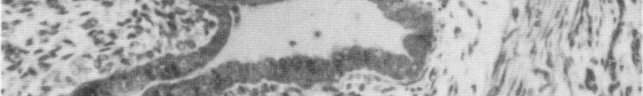

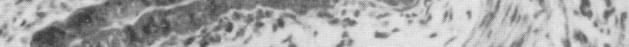
+

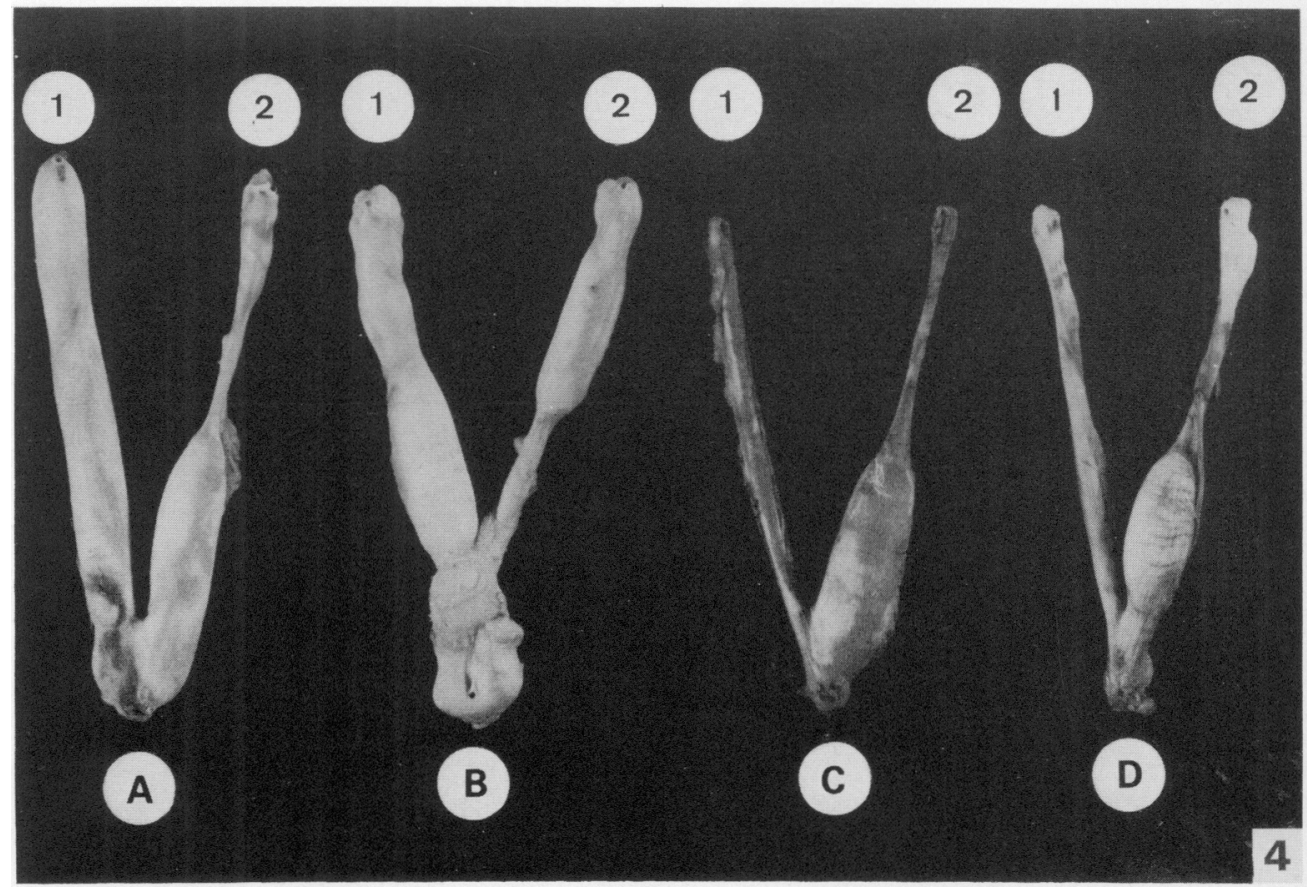


inhibitor of PG synthetase) given systemically to pregnant does inhibits increased capillary permeability at implantation sites (Hoffman, Di Pietro \& McKenna, 1978). In the rat also, PGF-2 $\alpha$ instilled into the uterine lumen of hormonally treated prepubertal animals is able to induce decidualization (Sananes. Baulieu \& Legoascogne, 1976). However, a DCR was not obtained when PGE-2. PGF-2 $\alpha$ or arachidonic acid was injected into uteri of pseudopregnant adult rats (Tobert. 1976). We also failed to induce a DCR by injection of PGs after removal of the epithelium (Group 8). However, half the amount of lipid-soluble drugs injected intraluminally into a ligated uterus is rapidly lost from the uterus (Conner \& Miller, 1973). In the rat. indomethacin inhibits the DCR when injected as late as $8 \mathrm{~h}$ after the inductive stimulus, suggesting that a prolonged synthesis of $\mathrm{PG}$ is necessary for proper stimulation.

In the rat, freezing of the uterine epithelium at -15 to $-25^{\circ} \mathrm{C}$ does not cause histologically apparent damage but decidualization and implantation were prevented (Ferrando \& Nalbandov, 1968). The block of these processes was overcome by intrauterine instillation of histamine. Histamine treatment in our experiments had no effect on decidualization after removal of the epithelium (Group 7), and the importance of the epithelium as an intermediate transmitter is again shown.

The importance of topographical relationships between the epithelium and stroma is confirmed by the fact that detachment of the epithelium without removal precludes the DCR (Group 4). Moreover, when a DCR was induced by scratching one half of a horn in which the epithelium had been removed from the other half, the DCR never extended from the intact half to the de-epitheliated segment (Pl. 1. Fig. 3). This suggests that the influence of the stimulated epithelium is locally restricted to the underlying stromal cells, without longitudinal transmission of the message which, as discussed above. would travel along the epithelium.

Our results are therefore compatible with, but do not confirm, both of the hypotheses for explaining the mechanism of decidualization: (1) synthesis and sustained release towards the stroma. of some labile (?) deciduogenic substance(s) produced by the luminal epithelium. (2) a combination, at the level of the epithelium. of a rapidly spreading membrane phenomenon (depolarization. change in permeability) associated with the release of deciduogenic substances. For both hypotheses, the chain of events involved at the level of the stroma would occur in a locally restricted fashion.

This work was supported by a grant from the Belgian F.R.S.M. L.B. and L.F. are Aspirant and Chercheur Quaifie at the Belgian F.N.R.S., respectively.

\section{References}

Ansbro, J.F. \& Schwartz, N.B. (1965) Use of electrical stimulation to elicit DCR in pseudopregnant rats. Phvsiologist, Wash. 8, 101-108.

Bitton-Casimiri, V., Rath, N.C. \& Psychoyos, A. (1977) A simple method for separation and culture of rat uterine epithelial cells. J. Endocr. 73. 537-538.

Conner, E.A. \& Miller, J.W. (1973) The distribution of selected substances into rat luminal fluid. J. Pharm. exp. Thér. 184. 291-298.

De Feo, V.J. (1967) Decidualization. In Cellular Biology of the Uterus, pp. 191-290. Ed. R. M. Wynn. North Holland Publishing Co., Amsterdam.

Fainstat, T. (1963) Extracellular studies of the uterus. Disappearance of the discrete collagen bundles in endometrial stroma during various reproductive states in the rat. Am. J. Anat. 112.337-369.

Ferrando, G. \& Nalbandov, A.V. (1968) Relative importance of histamine and estrogen on implantation in rats. Endocrinology 83, 933-937.

Finn, C.A. (1977) The implantation reaction. In Biology of the Uterus, pp. 245-308. Ed. R. M. Wynn. Plenum Press, New York.

Finn, C.A. \& Martin, L. (1974) The control of implantation. J. Reprod. Fert. 39, 195-206.

Finn, C.A. \& Porter, D.G. (1975) The decidual reaction. In The Uterus, pp. 74-85. Elek Science, London.

Hoffman, L.N., Strong, G.B., Davenport, G.R. \& Frolich, J.C. (1977) Deciduogenic effect of prostaglandins in the pseudopregnant rabbit. $J$. Reprod. Fert. 50, 231-237.

Hoffman, L.H., Di Pietro, D.L. \& McKenna, T.J. (1978) Effects of indomethacin on uterine capillary permeability and blastocyst development in rabbits. Prostaglandins 15, 823-828. 
Krebiehl, R.H. (1937) Cytological studies of the decidual reaction in the rat during early pregnancy and in the production of deciduomata. Physiol. Zool. 10, 212-234.

Pearse, A.G.E. (1968) Histochemistry, 3rd edn, Vol. 1, p. 583. Churchill Ltd, London.

Psychoyos, A. (1973) Hormonal control of implantation. Vitams Horm. 31, 201-256.

Sananes, N., Baulieu, E.E., Legoascogne, C. (1976)
Prostaglandin(s) as inductive factor of decidualization in the rat uterus. Molec. cell. Endocr. 6, 153-158.

Tobert, J.A. (1976) A study of the possible role of prostaglandins in decidualization using a non-surgical method for the instillation of fluids into the rat uterine lumen. J. Reprod. Fert. 47, 391-393.

Vokaer, A. \& Leroy, F. (1962) Experimental study on local factors in the process of ovo-implantation in the rat. Am. J. Obstet. Gynec. 83, 141-148.

Received 23 June 1980 